
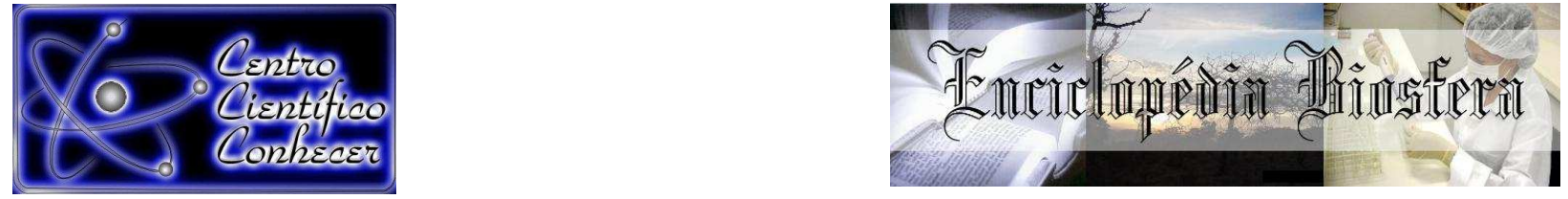

\title{
INFLUÊNCIA DO MÉTODO DE PROPAGAÇÃO E TIPOS DE RECIPIENTES NA QUALIDADE DE MUDAS DE Pinus maximinoi H. E. Moore
}

Fabrício Antônio Biernaski ${ }^{1}$, Antônio Carlos Nogueira², Evandro Vagner Tambarussi ${ }^{3}$, Ricardo Luis Mayer Weber ${ }^{4}$, James Stahl ${ }^{5}$

${ }^{1}$ Eng. Florestal, Mestre em Engenharia Florestal, Pesquisador na KLABIN S/A, Telêmaco Borba, PR-Brasil. (fbiernaski@klabin.com.br).

2 Professor Doutor, Universidade Federal do Paraná, Curitiba, PR-Brasil.

${ }^{3}$ Professor Doutor, Universidade Estadual do Centro-Oeste, Irati, PR-Brasil.

${ }^{4}$ Biólogo, Doutor em Biologia, Pesquisador na KLABIN S/A, Telêmaco Borba, PR-

Brasil.

${ }^{5}$ Eng. Florestal, Mestre em Produção Vegetal, Pesquisador na KLABIN S/A, Telêmaco Borba, PR-Brasil.

Recebido em: 02/10/2017 - Aprovado em: 21/11/2017 - Publicado em: 05/12/2017 DOI: 10.18677/EnciBio_2017B37

\section{RESUMO}

O Pinus maximinoi $\mathrm{H}$. E. Moore é uma espécie tropical com grande potencial para utilização em plantios comerciais de larga escala, entretanto não vem sendo amplamente explorada no Brasil devido a exigências silviculturais específicas, entre elas, a produção de mudas de qualidade. Na presente pesquisa objetivou-se avaliar os efeitos do uso de diferentes recipientes na qualidade de mudas de Pinus maximinoi produzidas via sementes e via enraizamento de miniestacas, instalandose um experimento fatorial com três fatores: (1) Método de Propagação (via sementes e via enraizamento de miniestacas), (2) Tipo de Recipientes (tubetes plásticos de $55 \mathrm{~cm}^{3}, 95 \mathrm{~cm}^{3}$ e $115 \mathrm{~cm}^{3}$ ) e (3) Aberturas Laterais (ausência e presença de aberturas laterais nos recipientes, para a poda aérea de raízes). Aos 230 dias as mudas foram avaliadas quanto ao seu diâmetro, altura, peso seco da parte aérea e de raízes, número de raízes e sobrevivência, assim como relações entre alguns destes fatores. As mudas produzidas por enraizamento de miniestacas apresentaram valores superiores em diâmetro, altura e pesos secos, porém inferiores em sobrevivência e número de raízes quando comparadas com mudas produzidas via sementes. A utilização de recipientes maiores influenciou positivamente o desenvolvimento das mudas, apresentando maior influência em mudas produzidas via enraizamento de miniestacas. A presença de aberturas laterais nos recipientes afetou a maioria dos parâmetros morfológicos avaliados, apresentando em geral valores inferiores para as características de crescimento, quando comparados à ausência de aberturas laterais.

PALAVRAS-CHAVE: miniestacas; raízes; volume de recipientes. 


\title{
INFLUENCE OF PROPAGATION METHOD AND CONTAINER TYPE ON PinUS maximinoi $H$. E. Moore SEEDLINGS
}

\begin{abstract}
Pinus maximinoi $\mathrm{H}$. E. Moore is a tropical pine with significant potential for use in large-scale commercial plantations. However, due to specific management requirements, especially the production of good quality seedlings, the species has not been widely exploited in Brazil. The aiming of this study was to evaluate the use of different containers on $P$. maximinoi plants produced from seeds and cuttings. We used a factorial experiment with three factors: (1) propagation method (seedlings vs. cuttings); (2) volume of container $\left(55 \mathrm{~cm}^{3}, 95 \mathrm{~cm}^{3}\right.$, and $\left.115 \mathrm{~cm}^{3}\right)$; and (3) the presence of lateral openings in containers for root pruning. After 230 days, the trials were evaluated for diameter, height, dry weight of shoots and roots, number of roots and survival. The relationships among the factors were analyzed too. The highest values for diameter, height, and dry weights, were found out in plants produced from cuttings, and lower values for survival and number of roots. Larger containers improve the development of the plants, with higher impact on cuttings. The presence of lateral openings negatively affected most of the evaluated growth parameters when compared with containers without lateral openings.
\end{abstract}

KEYWORDS: minicuttings; roots; container volume.

\section{INTRODUÇÃO}

O Brasil possui um total de 7,80 milhões de hectares de plantios florestais, sendo 1,58 milhões de hectares compostos pelo gênero Pinus spp., dentre os quais $42 \%$ estão localizados no Estado do Paraná (IBÁ, 2016). A espécie de pinus mais plantada no Paraná é o Pinus taeda L., entretanto de acordo com zoneamento climático da Embrapa Florestas, o Norte do Estado não está classificado como uma região adequada para o desenvolvimento da espécie, uma vez que a região está situada em um ambiente de transição entre clima subtropical e tropical (HIGA et al., 2008). Neste sentido, espécies de pinus tropicais apresentam grande potencial de utilização para o estabelecimento de povoamentos florestais mais produtivos na região, dentre elas o Pinus maximinoi.

A espécie Pinus maximinoi, além de apresentar rápido crescimento volumétrico, possui madeira de qualidade para diversas utilizações em produtos sólidos e para produção de celulose e papel (DVORAK et al., 2000; MATOS et al., 2012). Entretanto, ainda não vem sendo utilizada para estabelecimento de povoamentos comerciais em larga escala no Brasil, sendo que as primeiras tentativas sofreram entraves, principalmente devido à baixa sobrevivência dos povoamentos, relacionado a exigências diferenciadas na silvicultura da espécie, como por exemplo na produção de mudas com parâmetros de qualidade diferenciados, evitando especialmente a compactação de raízes nos recipientes. A espécie também exige maiores cuidados no preparo de solo, adubações e controle de plantas competidoras. Pinus maximinoi produz baixas quantidades de sementes, iniciando produção adequada somente em idades avançadas, tornando-se importante o desenvolvimento de métodos de propagação vegetativa para viabilizar a produção de mudas provenientes de materiais genéticos superiores.

Neste sentido, a produção de mudas via enraizamento de miniestacas vem sendo utilizada em diversas espécies de pinus, sendo que estudos comparativos demonstram equivalência em crescimento volumétrico em campo quando são utilizados propágulos juvenis (CUMBIE et al., 2011; LU et al., 2012; BONGA, 2015). 
Por outro lado, a utilização de diferentes tipos de recipientes para produção de mudas de pinus pode afetar a qualidade das mudas, podendo influenciar a sobrevivência e o desenvolvimento das árvores em campo (PUÉRTOLAS et al., 2012; JELIC, 2014).

Desta forma, o presente estudo objetivou avaliar os efeitos do uso de diferentes recipientes na qualidade de mudas de Pinus maximinoi produzidas via sementes e via enraizamento de miniestacas.

\section{MATERIAL E MÉTODOS}

Foram testados os efeitos do uso de diferentes recipientes na qualidade de mudas de Pinus maximinoi produzidas via sementes e via enraizamento de miniestacas. Instalou-se um experimento fatorial com três fatores: (1) Método de Propagação via sementes e via enraizamento de miniestacas; (2) Tipo de Recipientes, utilizando tubetes plásticos de $55 \mathrm{~cm}^{3}, 95 \mathrm{~cm}^{3}$ e $115 \mathrm{~cm}^{3}$; e (3) Ausência ou presença de Aberturas Laterais nos recipientes, para a poda aérea de raízes (Tabela 1 ).

TABELA 1 - Relação de tratamentos aplicados na produção de mudas de Pinus maximinoi aos 230 dias.

\begin{tabular}{|c|c|c|}
\hline Método de Propagação & Tipo de Recipiente & Aberturas Laterais \\
\hline \multirow{4}{*}{ Enraizamento de miniestacas } & $55 \mathrm{~cm}^{3}$ & Ausência \\
\cline { 2 - 3 } & \multirow{2}{*}{$95 \mathrm{~cm}^{3}$} & Presença \\
\cline { 2 - 3 } & \multirow{2}{*}{$115 \mathrm{~cm}^{3}$} & Ausência \\
\cline { 2 - 3 } & \multirow{2}{*}{$55 \mathrm{~cm}^{3}$} & Presença \\
\cline { 2 - 3 } & \multirow{2}{*}{ Sementes } & Pusência \\
\cline { 2 - 3 } & \multirow{2}{*}{$95 \mathrm{~cm}^{3}$} & Ausênça \\
\cline { 2 - 3 } & \multirow{2}{*}{$115 \mathrm{~cm}^{3}$} & Presença \\
\cline { 2 - 3 } & & Ausência \\
\cline { 2 - 3 } & & Presença \\
\cline { 2 - 3 } & & Presença \\
\hline
\end{tabular}

O experimento foi instalado em maio de 2014 no viveiro de pesquisa da

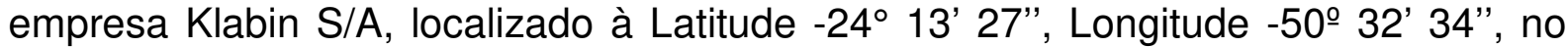
município de Telêmaco Borba, Paraná, Brasil. O local está a 890 metros de altitude e possui temperatura média anual de $19,5{ }^{\circ} \mathrm{C}$ e precipitação média anual de 1600 mm (KLABIN, 2017), com clima Cfa segundo classificação climática de Köppen.

Para viabilizar a comparação entre os métodos de propagação, foi utilizado o mesmo material genético para os tratamentos propagados via sementes e via enraizamento de miniestacas, sendo composto por um único lote de sementes contendo cinco progênies de polinização aberta de Pinus maximinoi.

O substrato utilizado foi o mesmo para todos os tratamentos e foi formulado com $50 \%$ de casca de pinus decomposta, $30 \%$ de casca de arroz carbonizada e $20 \%$ de fibra de coco, acrescentando adubação de base de liberação lenta e de disponibilidade imediata.

Durante o desenvolvimento das mudas foram utilizadas irrigações com sistema de aspersão e fertilizações diárias (iniciando aos 60 dias) com soluções líquidas de macro e micronutrientes, sendo que todos os tratamentos receberam o mesmo manejo de água e nutrição, seguindo o protocolo padrão de produção de mudas do viveiro. 
Devido a exigências de manejo distintas no início da produção de mudas via sementes e via enraizamento de miniestacas, primeiramente o experimento foi dividido em dois, de acordo com o método de propagação. Após o enraizamento das miniestacas e a obtenção de plântulas germinadas (60 dias), todos os tratamentos foram agrupados em casa de sombra, onde receberam o delineamento experimental definitivo.

\section{Produção de mudas via enraizamento de miniestacas}

Mudas de quatro meses de idade provenientes de sementes de um lote composto por cinco progênies de polinização aberta de Pinus maximinoi foram plantadas em canaletas de areia sob estufa agrícola com cobertura plástica e cultivados em sistema semi-hidropônico, realizando-se podas sucessivas para formação de minicepas (minijardim). Adubações diárias com solução nutritiva de macro e micronutrientes foram aplicadas por sistema de gotejamento. Após 22 meses, miniestacas de aproximadamente $7 \mathrm{~cm}$ de comprimento e $2 \mathrm{~mm}$ de diâmetro foram coletadas das minicepas com auxílio de tesouras, acondicionadas em caixas de isopor e borrifadas com água para manutenção da umidade. Em seguida as bases das miniestacas foram inseridas aproximadamente $2,5 \mathrm{~cm}$ de profundidade no substrato, em seis diferentes tipos de recipientes (Tabela 1) e acondicionados em bandejas. O substrato, fertilização de base e fertirrigação (início após 60 dias) foram idênticos para todos os tratamentos, sendo os mesmos utilizados na produção de mudas via sementes.

O material foi imediatamente acondicionado em casa de vegetação com irrigação por micro aspersão, com umidade relativa média do ar de $80 \%$ e temperaturas variando entre 20 e $30^{\circ} \mathrm{C}$. Após permanência por 60 dias em casa de vegetação para formação de raízes as bandejas foram acondicionadas em casa de sombra coberta com tela de $50 \%$ de sombreamento.

\section{Produção de mudas via sementes}

Sementes de um lote composto por cinco progênies de polinização aberta de Pinus maximinoi (mesmo lote utilizado para produção de minicepas e miniestacas) foram plantadas em seis diferentes tipos de recipientes contendo substrato, conforme os tratamentos definidos (Tabela 1). As bandejas contendo os recipientes foram acondicionadas em estufa agrícola com irrigação via micro aspersão, para germinação das sementes.

O substrato, fertilização de base e fertirrigação (início após 60 dias) foram idênticos para todos os tratamentos, sendo os mesmos utilizados na produção de mudas via enraizamento de miniestacas. Após 60 dias as bandejas foram transferidas para casa de sombra coberta com tela de $50 \%$ de sombreamento.

\section{Agrupamento no delineamento estatístico definitivo}

Durante o período de permanência em casa de sombra, após obtenção definitiva de plântulas via sementes e após o enraizamento definitivo das miniestacas, todos os 12 tratamentos receberam o delineamento experimental final, distribuídos em blocos ao acaso, 6 repetições e 36 plantas por parcela.

Os tratamentos permaneceram por 60 dias em casa de sombra e em seguida as bandejas foram acondicionadas em pátio a pleno sol para crescimento e aclimatação, com irrigação por aspersão, conforme a necessidade. Após permanecer por 110 dias em pátio a pleno sol realizou-se a avaliação final do experimento (mudas com 230 dias de idade a partir da semeadura e estaqueamento). 


\section{Avaliações e análise de dados}

A avaliação do experimento foi realizada quando as mudas atingiram 230 dias, efetuando a mensuração de diâmetro de colo (DIAM) com paquímetro digital e altura com régua (ALT). Os dados foram tabulados e obtidos os valores médios por tratamento, em seguida três plantas com altura média $(17,3 \mathrm{~cm})$ foram obtidas aleatoriamente de cada parcela, para as avaliações destrutivas. As mudas foram retiradas dos recipientes e em seguida as raízes foram lavadas delicadamente com água para retirada completa do substrato.

Foi realizada a contagem do número de raízes saindo do eixo principal de cada muda (№ RAÍZES) e em seguida cada muda foi seccionada na região do colo, separando a parte aérea das raízes, sendo as mesmas acondicionadas em sacos de papel e secas em estufa elétrica de circulação forçada até peso constante, para obtenção do peso seco da parte aérea (PSA), peso seco de raízes (PSR) e peso seco total (PST). Variáveis adicionais foram criadas com a divisão da altura pelo diâmetro do colo $(H / D)$ e pela divisão do peso seco da parte aérea pelo peso seco de raízes (PSA/PSR). O percentual de mortalidade de mudas de cada parcela também foi mensurado ( $\%$ MORT).

A análise de dados foi efetuada utilizando o software SISVAR $₫$ (FERREIRA, 2014), realizando análises de variância em fatorial triplo e testes de comparação de médias pelo teste de Tukey a $5 \%$ de probabilidade.

\section{RESULTADOS E DISCUSSÃO}

A análise de variância evidenciou diferenças significativas ao analisar cada fator separadamente. O fator Método de Propagação apresentou diferenças significativas $(p<0,01)$ nas nove variáveis analisadas, seguido pelo fator Tipo de Recipiente (diferenças em seis variáveis) e fator Aberturas Laterais (diferenças em cinco variáveis). Interações significativas foram identificadas entre os fatores Método de Propagação e Tipo de Recipiente para cinco variáveis, enquanto duas variáveis apresentaram interações entre os fatores Tipos de Recipientes e Abertura Lateral (Tabela 2).

TABELA 2 - Tabela resumo de $p$ valor da análise de variância em esquema fatorial triplo, considerando as variáveis avaliadas na produção de mudas de Pinus maximinoi aos 230 dias.

\begin{tabular}{|c|c|c|c|c|c|c|c|c|c|c|}
\hline & & DIAM & ALT & $H / D$ & PSR & PSA & PST & PSA/PSR & $\mathrm{N}^{0}$ RAIIZES $^{(1)}$ & $\%$ MORT $^{(1)}$ \\
\hline FV & GL & $\mathrm{Pr}>\mathrm{Fc}$ & $\mathrm{Pr}>\mathrm{Fc}$ & $\mathrm{Pr}>\mathrm{Fc}$ & $\mathrm{Pr}>\mathrm{Fc}$ & $\mathrm{Pr}>\mathrm{Fc}$ & $\mathrm{Pr}>\mathrm{Fc}$ & $\mathrm{Pr}>\mathrm{Fc}$ & $\mathrm{Pr}>\mathrm{Fc}$ & $\mathrm{Pr}>\mathrm{Fc}$ \\
\hline PROPAGAÇÃO & 1 & $0,0000^{\star \star}$ & $0,0000^{* *}$ & $0,0000^{* *}$ & $0,0018^{\star \star}$ & $0,0000^{* *}$ & $0,0000^{* \star}$ & $0,0000^{\star \star}$ & $0,0000^{* *}$ & $0,0098^{* *}$ \\
\hline RECIPIENTE & 2 & $0,0187^{*}$ & $0,0000^{* *}$ & $0,0000^{* *}$ & $0,0006^{\star \star}$ & $0,0000^{* *}$ & $0,0000^{* \star}$ & $0,9978 \mathrm{~ns}$ & $0,1069 n s$ & $0,0746 n s$ \\
\hline ABERTURA & 1 & $0,0033^{\star *}$ & $0,0004^{* *}$ & $0,1542 \mathrm{~ns}$ & $0,0000^{* *}$ & $0,6585 \mathrm{~ns}$ & $0,0341^{* \star}$ & $0,0000^{\star *}$ & $0,2555 \mathrm{~ns}$ & $0,5039 \mathrm{~ns}$ \\
\hline PROPAGAÇÃO^RECIPIENTE & 2 & $0,3593 \mathrm{~ns}$ & $0,0002^{* *}$ & $0,0103^{*}$ & $0,0012^{* *}$ & $0,0002^{\star \star}$ & $0,0001^{\star *}$ & $0,7038 \mathrm{~ns}$ & $0,3159 \mathrm{~ns}$ & $0,0899 \mathrm{~ns}$ \\
\hline PROPAGAÇÃO*ABERTURA & 1 & $0,1859 \mathrm{~ns}$ & $0,1246 \mathrm{~ns}$ & $0,6254 n s$ & $0,0555 \mathrm{~ns}$ & $0,5863 \mathrm{~ns}$ & $0,2760 \mathrm{~ns}$ & $0,0540 \mathrm{~ns}$ & $0,7292 \mathrm{~ns}$ & $0,6259 \mathrm{~ns}$ \\
\hline RECIPIENTE*ABERTURA & 2 & $0,1642 \mathrm{~ns}$ & $0,5545 \mathrm{~ns}$ & $0,3853 \mathrm{~ns}$ & $0,0049^{* *}$ & $0,1444 n s$ & $0,0305^{*}$ & $0,4452 \mathrm{~ns}$ & $0,0532 \mathrm{~ns}$ & $0,5718 \mathrm{~ns}$ \\
\hline PROPAGAÇÃO*RECIPIENTE*ABERTURA & & $0,6526 n s$ & $0,9541 \mathrm{~ns}$ & $0,6108 \mathrm{~ns}$ & $0,2730 \mathrm{~ns}$ & $0,4908 \mathrm{~ns}$ & $0,4362 \mathrm{~ns}$ & $0,1216 \mathrm{~ns}$ & $0,9318 \mathrm{~ns}$ & $0,9317 \mathrm{~ns}$ \\
\hline BLOCO & 5 & $0,0042^{*}$ & $0,0000^{\star \star}$ & $0,0001^{\text {** }}$ & $0,3646 \mathrm{~ns}$ & $0,8205 \mathrm{~ns}$ & $0,9222 \mathrm{~ns}$ & $0,2675 \mathrm{~ns}$ & 0,9930 ns & $0,1472 \mathrm{~ns}$ \\
\hline $\mathrm{CV}(\%)$ & & 6,82 & 8,02 & 7,11 & 19,27 & 18,34 & 16,35 & 19,46 & 10,02 & 3,39 \\
\hline Média geral: & & 3,03 & 17,28 & 5,89 & 1,71 & 4,24 & 5,94 & 2,54 & 2,57 & 0,72 \\
\hline $\begin{array}{l}\text { GL erro } \\
\text { GL Total corrigido }\end{array}$ & $\begin{array}{l}55 \\
71\end{array}$ & & & & & & & & & \\
\hline
\end{tabular}

DIAM: diâmetro do colo $(\mathrm{mm})$; ALT: altura $(\mathrm{cm})$; H/D: relação altura/diâmetro; PSR: peso seco de raiz (g); PSA: peso seco da parte aérea (g); PST: peso seco total (g); PSA/PSR: relação peso seco aéreo/ peso seco de raiz; ${ }^{(1)}$ dados transformados: № RAíZES: raiz quadrada do número de raízes; \% MORT: raiz quadrada de porcentagem de mortalidade de mudas + 0,5.

FV: fonte de variação; GL: graus de liberdade; CV: coeficiente de variação.

${ }^{* *}$ significativo a $1 \%$ de probabilidade; *significativo a $5 \%$ de probabilidade; ns não significativo a $5 \%$ de probabilidade. 
É possível identificar diferenças significativas para as variáveis que não apresentaram interações entre os fatores (Tabela 3). Não foram identificadas interações entre nenhum dos três fatores ao analisar as variáveis: diâmetro do colo (DIAM), relação peso seco aéreo/ peso seco de raízes (PSA/PSR), número de raízes (№ RAÍZES) e percentual de mortalidade (\% MORT). Ao analisar as variáveis altura $(A L T)$, relação altura/diâmetro $(H / D)$ e peso seco aéreo (PSA) não foram identificadas interações com o fator Aberturas Laterais.

TABELA 3 - Comparação de médias utilizando teste de Tukey a 5\% de probabilidade para as variáveis sem interação significativa entre os fatores analisados na produção de mudas de Pinus maximinoi aos 230 dias.

\begin{tabular}{|c|c|c|c|c|c|c|c|}
\hline & & \multicolumn{2}{|c|}{ DIAM (mm) } & \multicolumn{2}{|c|}{ PSA/PSR } & \multicolumn{2}{|c|}{ No RAIIZES } \\
\hline \multirow{2}{*}{ Propagação } & Miniestaca & 3,55 & $a$ & 3,15 & $a$ & 3,52 & $b$ \\
\hline & Semente & 2,50 & $b$ & 1,93 & $b$ & 10,82 & a \\
\hline \multirow{3}{*}{ Recipiente } & $55 \mathrm{~cm}^{3}$ & 2,93 & $b$ & 2,54 & a & 7,33 & $a$ \\
\hline & $95 \mathrm{~cm}^{3}$ & 3,05 & $a b$ & 2,54 & $a$ & 7,43 & a \\
\hline & $115 \mathrm{~cm}^{3}$ & 3,11 & a & 2,53 & a & 6,75 & a \\
\hline \multirow{3}{*}{$\begin{array}{l}\text { Aberturas } \\
\text { laterais }\end{array}$} & Com & 2,95 & $b$ & 2,84 & $a$ & 6,99 & a \\
\hline & Sem & 3,10 & a & 2,24 & $b$ & 7,35 & a \\
\hline & & \multicolumn{2}{|c|}{ ALT $(\mathrm{cm})$} & $\mathrm{H} / \mathrm{D}$ & & \multicolumn{2}{|c|}{ PSA (g) } \\
\hline \multirow{2}{*}{$\begin{array}{l}\text { Aberturas } \\
\text { laterais }\end{array}$} & Com & 16,66 & $b$ & 5,82 & $a$ & 4,20 & a \\
\hline & Sem & 17,90 & a & 5,96 & a & 4,28 & a \\
\hline
\end{tabular}

DIAM: diâmetro do colo (mm); PSA/PSR: relação peso seco aéreo/ peso seco de raiz; № RAíZES: número de raízes; \% MORT: porcentagem de mortalidade de mudas; ALT: altura $(\mathrm{cm}) ; \mathrm{H} / \mathrm{D}$ : relação altura/diâmetro; PSA: peso seco da parte aérea (g);

Médias seguidas de mesma letra na vertical indicam que os tratamentos são estatisticamente iguais pelo teste de Tukey a $5 \%$ de probabilidade

Os fatores Método de Propagação e Tipo de Recipiente apresentaram interação significativa ao analisar as variáveis altura (ALT), relação altura/ diâmetro $(\mathrm{H} / \mathrm{D})$, peso seco de raiz (PSR), peso seco aéreo (PSA) e peso seco total (PST) (Tabela 4). 
TABELA 4 - Comparação de médias utilizando Teste de Tukey a 5\% de probabilidade para as variáveis que apresentaram interação significativa entre os fatores Tipo de Recipiente e Método de Propagação na produção de mudas de Pinus maximinoi aos 230 dias.

\begin{tabular}{|c|c|c|c|c|}
\hline \multirow[b]{2}{*}{ Recipiente } & \multicolumn{2}{|c|}{ ALT $(\mathrm{cm})$} & \multicolumn{2}{|c|}{$\mathrm{H} / \mathrm{D}$} \\
\hline & Miniestaca & Semente & Miniestaca & Semente \\
\hline $55 \mathrm{~cm}^{3}$ & $16,68 \mathrm{~B} \mathrm{a}$ & $14,67 \mathrm{~B} \mathrm{~b}$ & $4,87 \mathrm{~B} \mathrm{~b}$ & $6,23 \mathrm{~A}$ a \\
\hline $95 \mathrm{~cm}^{3}$ & $17,90 \mathrm{~B}$ a & $16,37 \mathrm{~A} \mathrm{~b}$ & $5,18 \mathrm{~B} \mathrm{~b}$ & $6,49 \mathrm{~A}$ a \\
\hline $115 \mathrm{~cm}^{3}$ & $21,43 \quad \mathrm{~A} a$ & $16,61 \mathrm{~A} \mathrm{~b}$ & $5,96 \mathrm{~A} \mathrm{~b}$ & $6,63 \mathrm{~A}$ a \\
\hline & \multicolumn{2}{|c|}{ PSR (g) } & \multicolumn{2}{|c|}{ PSA (g) } \\
\hline Recipiente & Miniestaca & Semente & Miniestaca & Semente \\
\hline $55 \mathrm{~cm}^{3}$ & 1,75 & $1,48 \quad \mathrm{~A} a$ & $4,97 \mathrm{~B}$ a & $2,87 \mathrm{~A} \mathrm{~b}$ \\
\hline $95 \mathrm{~cm}^{3}$ & 1,51 & $1,64 \quad \mathrm{~A}$ a & 4,80 B a & $3,02 \mathrm{~A} \mathrm{~b}$ \\
\hline $115 \mathrm{~cm}^{3}$ & 2,24 & $1,62 \quad \mathrm{~A} \mathrm{~b}$ & $6,70 \mathrm{~A}$ a & $3,05 \mathrm{~A} \mathrm{~b}$ \\
\hline
\end{tabular}

\begin{tabular}{lllll}
\hline & \multicolumn{4}{c}{ PST $(\mathrm{g})$} \\
\cline { 2 - 5 } Recipiente & \multicolumn{2}{c}{ Mropagação } & \multicolumn{3}{c}{ Miniestaca } & Semente \\
\hline $55 \mathrm{~cm}^{3}$ & 6,71 & B a & 4,36 & A b \\
$95 \mathrm{~cm}^{3}$ & 6,31 & B a & 4,66 & A b \\
$115 \mathrm{~cm}^{3}$ & 8,95 & A a & 4,67 & A b \\
\hline
\end{tabular}

ALT: altura (cm); H/D: relação altura/diâmetro; PSR: peso seco de raiz (g); PSA: peso seco da parte aérea (g); PST: peso seco total (g).

Médias seguidas de mesma letra maiúscula na vertical são estatisticamente iguais e médias seguidas de mesma letra minúscula na horizontal são estatisticamente iguais, pelo teste de Tukey a $5 \%$ de probabilidade.

O fator Tipo de Recipiente também apresentou interação significativa com o fator Aberturas Laterais para as variáveis PSR e PST (Tabela 5).

TABELA 5 - Comparação de médias utilizando teste de Tukey a 5\% de probabilidade para as variáveis que apresentaram interação significativa entre os fatores Tipo de Recipiente e Abertura Lateral na produção de mudas de Pinus maximinoi aos 230 dias.

\begin{tabular}{|c|c|c|c|c|}
\hline \multirow[b]{2}{*}{ Recipiente } & \multicolumn{2}{|c|}{ PSR (g) } & \multicolumn{2}{|c|}{ PST (g) } \\
\hline & $\begin{array}{l}\text { Sem } \\
\text { abertura }\end{array}$ & $\begin{array}{l}\text { Com } \\
\text { abertura }\end{array}$ & $\begin{array}{l}\text { Sem } \\
\text { abertura }\end{array}$ & $\begin{array}{l}\text { Com } \\
\text { abertura }\end{array}$ \\
\hline $55 \mathrm{~cm}^{3}$ & $1,92 \mathrm{~A} a$ & $1,31 \mathrm{~B} \quad \mathrm{~b}$ & $6,08 \mathrm{~B}$ a & $4,99 \mathrm{~B}$ \\
\hline $95 \mathrm{~cm}^{3}$ & $1,60 \mathrm{~B}$ a & $1,55 \mathrm{AB}$ a & 5,30 B a & $5,67 \mathrm{AB}$ \\
\hline $115 \mathrm{~cm}^{3}$ & $2,23 \mathrm{~A}$ a & $1,63 \mathrm{~A} \quad \mathrm{~b}$ & $7,19 \mathrm{~A}$ a & $6,43 \mathrm{~A}$ \\
\hline
\end{tabular}

PSR: peso seco de raiz (g); PST: peso seco total $(\mathrm{g})$.

Médias seguidas de mesma letra maiúscula na vertical são estatisticamente iguais e médias seguidas de mesma letra minúscula na horizontal são estatisticamente iguais, pelo teste de Tukey a $5 \%$ de probabilidade.

\section{Fator Método de Propagação}

A variável DIAM apresentou diferenças significativas entre os métodos de propagação, sendo que as mudas propagadas via enraizamento de miniestacas 
apresentaram maiores diâmetros de colo $(3,55 \mathrm{~mm})$ enquanto as propagadas via sementes apresentaram $2,50 \mathrm{~mm}$ (Tabela 3). As mudas produzidas por enraizamento de miniestacas também apresentaram valores estatisticamente superiores em ALT, PSA e PST, comparativamente às mudas produzidas via sementes (Tabela 4). Resultados semelhantes foram relatados por Bordin et al., (2005) em Malpighia emarginata D.C., sendo que mudas produzidas por enraizamento de estacas apresentaram maiores valores de diâmetro do colo, altura e peso da parte aérea.

Para PSR somente houve diferenças nos tratamentos com recipientes de 115 $\mathrm{cm}^{3}$, com superioridade das mudas propagadas por enraizamento de miniestacas (Tabela 4). Jesus et al., (2006) trabalhando com Coffea arabica L., em recipientes plásticos de $10 \mathrm{~cm} \times 20 \mathrm{~cm}$, também verificaram que mudas produzidas por estaquia apresentam maior peso seco de raízes comparativamente à mudas produzidas via sementes.

Observou-se que estas diferenças devem estar relacionadas ao fato das mudas produzidas via miniestacas terem iniciado o processo de desenvolvimento a partir de miniestacas com aproximadamente $2 \mathrm{~mm}$ de diâmetro e $4,5 \mathrm{~cm}$ de altura (7 $\mathrm{cm}$ de miniestaca, sendo $2,5 \mathrm{~cm}$ inseridos no substrato). Embora as miniestacas tiveram que desenvolver raízes para seu crescimento, as mudas produzidas via germinação de sementes não se desenvolveram tão rapidamente quando comparadas às mudas de miniestaquia.

Considerando a variável PSA/PSR, a relação mais equilibrada foi obtida pelo método de propagação via sementes $(1,93)$ enquanto a propagação via enraizamento de miniestacas apresentou relação de $(3,15)$, estatisticamente diferente da primeira (Tabela 3).

O número médio de raízes desenvolvidas a partir do eixo principal da muda foi estatisticamente superior nas mudas produzidas via sementes $(10,82)$, enquanto mudas propagadas via enraizamento de miniestacas apresentaram 3,52 raízes principais em média (Tabela 03).

Embora tenham sido verificadas tais diferenças entre os métodos de propagação, faz-se necessário avaliar também o seu efeito no desenvolvimento das árvores em campo. Cumbie et al. (2011) não identificaram diferenças de crescimento de Pinus taeda aos 10 anos quando comparou a utilização de mudas produzidas via propagação vegetativa e via germinação de sementes. Nesta mesma linha, Mora et al. (2005) encontrou resultados similares em Pinus radiata aos sete anos, assim como LU et al. (2012) avaliando Pinus banksiana aos oito anos.

A relação $H / D$ foi mais favorável para nas mudas produzidas via enraizamento de miniestacas, apresentando valores menores que as mudas produzidas via sementes (Tabela 4). Bayley e Kietzka (1996) verificaram que mudas de Pinus patula com menores relações H/D apresentam maior sobrevivência em campo.

O percentual de mortalidade foi maior nas mudas propagadas via enraizamento de miniestacas $(2,70 \%)$, estatisticamente superior às mudas propagadas via sementes $(0,39 \%)$. A maior mortalidade das mudas produzidas via enraizamento de miniestacas deve ter ocorrido pela má formação de raízes em parte das mudas propagadas por este método, que após a saída da casa de vegetação não resistiram o ambiente externo à pleno sol (Tabela 3).

\section{Fator Tipo de Recipiente}

A variável diâmetro do colo (DIAM) não apresentou interação entre os fatores, sendo que independentemente do Método de Propagação e das Aberturas Laterais, 
os melhores resultados foram observados nos recipientes maiores de $115 \mathrm{~cm}^{3}(3,11$ $\mathrm{mm})$ e $95 \mathrm{~cm}^{3}(3,05 \mathrm{~mm})$ sendo estatisticamente iguais, enquanto o recipiente de 55 $\mathrm{cm}^{3}$ apresentou o menor diâmetro $(2,93 \mathrm{~mm})$, porém estatisticamente igual ao recipiente $95 \mathrm{~cm}^{3}$ (Tabela 3 ).

Analisando as variáveis PSA/PSR, № RAÍZES e \% MORT, não foram identificadas interações entre os três fatores avaliados, nem foram observadas diferenças significativas entre os tipos de recipientes (Tabela 3).

Observando em conjunto os resultados do fator Tipo de Recipientes (Tabelas

3 e 4), percebe-se que as variáveis de crescimento DIAM e ALT são impactadas positivamente com o aumento do volume dos recipientes para os dois métodos de propagação. Entretanto, as variáveis peso seco (PSR, PSA, PST) foram influenciadas pelo volume dos recipientes somente nas mudas de enraizamento de miniestacas.

Para as mudas produzidas via enraizamento de miniestacas, analisando as variáveis $A L T, H / D, P S R, P S A$ e PST, o uso de recipientes de $115 \mathrm{~cm}^{3}$ apresentou valores estatisticamente superiores para estas variáveis, quando comparado com os recipientes menores de $95 \mathrm{~cm}^{3}$ e $55 \mathrm{~cm}^{3}$ (Tabela 4).

Considerando a variável ALT dentro do fator propagação via sementes, os recipientes $95 \mathrm{~cm}^{3}$ e $115 \mathrm{~cm}^{3}$ apresentaram desempenho estatisticamente igual, resultando em mudas mais altas $(16,37 \mathrm{~cm}$ e $16,61 \mathrm{~cm}$, respectivamente), enquanto o tratamento $55 \mathrm{~cm}^{3}$ produziu mudas mais baixas $(14,67 \mathrm{~cm})$, estatisticamente inferior dos outros tamanhos de recipientes. Ainda, dentro do fator propagação via sementes, os três diferentes tamanhos de recipientes não provocaram diferenças significativas considerando as variáveis H/D, PSR, PSA e PST (Tabela 4).

As demais variáveis PSA/PSR, № RAÍZES e \% MORT não apresentaram interação entre os fatores, nem seus valores diferiram estatisticamente entre os diferentes tamanhos de recipientes.

Donald (1992) obteve resultados similares trabalhando com três volumes de recipientes em Pinus radiata (36, 66 e $80 \mathrm{~cm}^{3}$ ) apresentando melhores resultados para mudas de 7 meses produzidas nos recipientes maiores: 3,03; 3,56 e 3,57 mm de diâmetro do colo, 19,6; 23,2 e 24,8 cm de altura, 0,50; 0,72 e 1,11 $\mathrm{g}$ de massa de raízes e 1,$72 ; 2,53$ e 3,50 g de massa total, respectivamente.

Jelic (2014) testou diferentes tamanhos de recipientes para produção de mudas de Pinus pinea $\mathrm{L}$. e obteve mudas de melhor qualidade utilizando recipientes maiores, refletindo em crescimento superior em campo aos seis anos. Puértolas et al, (2012) trabalhando com Pinus halepensis e Pinus pinea obtiveram o mesmo padrão de resultados.

Ferraz e Engel (2011) também constataram que para Hymenaea courbaril L. var. stilbocarpa (Hayne) Lee et Lang., Tabebuia chrysotricha (Mart. ex DC.) Sandl.) e Parapiptadenia rigida (Benth.) Brenan o aumento do volume do tubete influenciou positivamente no desenvolvimento das mudas, proporcionando mudas com altura e diâmetro do colo superiores às produzidas em tubetes de volumes menores.

Tsakaldimi (2013) verificou que o diâmetro do colo e peso seco total de mudas de Pinus halepensis apresentaram correlações positivas com a sobrevivência inicial em campo. Segundo Dobner Júnior et al., (2013) mudas de Pinus taeda produzidas em recipientes de $200 \mathrm{~cm}^{3}$ apresentaram maior desenvolvimento de diâmetro e altura em viveiro comparadas com mudas produzidas em recipientes de $60 \mathrm{~cm}^{3}$. Após dois anos de plantio em campo as mudas maiores refletiram em alturas maiores de plantas, entretanto aos 9 anos não foram mais identificadas diferenças no volume das árvores ao avaliar os dois tratamentos aplicados. 
Considerando as raízes, Mitchell et al. (2005) testaram recipientes com diferentes volumes em Pinus patula, verificando que o tratamento com $125 \mathrm{~cm}^{3}$ de volume obteve maior massa de raízes e apresentou $27 \%$ de diferença em volume de madeira no campo aos sete anos, quando comparado ao recipiente de $80 \mathrm{~cm}^{3}$. Neste mesmo aspecto, Rose et al. (1991) verificaram em Pseudotsuga menziesii (Mirb.) Franco, que mudas com maior volume de raízes apresentaram desenvolvimento superior dois anos após o plantio em campo.

\section{Fator Aberturas Laterais}

O fator Aberturas Laterais dos recipientes não refletiu em diferenças significativas nas variáveis $N^{\circ}$ RAÍZES, \% MORT, relação H/D e PSA (Tabela 3).A presença de aberturas laterais refletiu em menores valores de diâmetro do colo (DIAM) das mudas $(2,95 \mathrm{~mm})$, estatisticamente inferior aos recipientes sem as aberturas laterais $(3,10 \mathrm{~mm})$. O mesmo ocorreu com a variável altura, com média de $16,66 \mathrm{~cm}$ para as mudas produzidas em recipientes com abertura e 17,90 nos recipientes sem aberturas laterais, sendo estatisticamente diferentes entre si (Tabela 3). Uma vez que todos os tratamentos receberam o mesmo manejo em viveiro, tal diferença em desenvolvimento das mudas pode estar relacionada a diferentes níveis de disponibilidade de água entre os tratamentos com ausência e presença de aberturas laterais, uma vez que aberturas laterais promovem maior aeração, diminuindo mais rapidamente a quantidade de água disponível no substrato. Lamhamedi et al., (2001) também observaram este efeito em mudas de Picea glauca produzidas em recipientes com aberturas laterais ao variar a quantidade de água aplicada.

A relação PSA/PSR foi mais equilibrada nas mudas produzidas em recipientes sem aberturas laterais $(2,24)$, sendo estatisticamente diferente do índice obtido no tratamento com aberturas laterais $(2,84)$ (Tabela 3 ).

O fator Abertura Lateral apresentou interação significativa com o Tipo de Recipiente. Para o recipiente com volume de $55 \mathrm{~cm}^{3}$ a ausência de aberturas laterais propiciou valores significativamente maiores de PSR e PST. Para o volume intermediário de $95 \mathrm{~cm}^{3}$ não houve diferenças entre ausência ou presença de aberturas laterais ao considerar as variáveis PSR e PST. No caso dos recipientes de $115 \mathrm{~cm}^{3}$, os maiores valores de PSR foram obtidos sem a utilização de aberturas laterais $(2,23 \mathrm{~g})$, enquanto a presença de aberturas laterais produziram 1,63 $\mathrm{g}$ de matéria seca, estatisticamente inferior. Ainda considerando os recipientes de 115 $\mathrm{cm}^{3}$, não houve diferença significativa na ausência ou presença de aberturas laterais ao analisar a variável PST (Tabela 5).

Ortega et al., (2006), avaliando produção de mudas de Pinus radiata, observaram resultados semelhantes, obtendo menor crescimento e menor produção de biomassa aérea e de raízes ao utilizar recipientes com aberturas laterais, comparativamente a recipientes sem aberturas laterais. $O$ fator presença de aberturas laterais apresentou o mesmo padrão de resultados em relação às variáveis PSR e PST, sendo que quanto maiores os recipientes, maiores os valores de peso seco obtidos, sendo estatisticamente distintos entre $55 \mathrm{~cm}^{3}$ e $115 \mathrm{~cm}^{3}$ (Tabela 5).

O fator ausência de aberturas laterais apresentou resultados complexos para as variáveis PSR e PST, sendo que os maiores valores foram encontrados para os recipientes maiores $\left(115 \mathrm{~cm}^{3}\right)$, seguidos pelos recipientes menores $\left(55 \mathrm{~cm}^{3}\right)$, sendo que os menores valores foram obtidos nos recipientes de volume intermediário (95 $\left.\mathrm{cm}^{3}\right)$ (Tabela 5). 
Embora os recipientes com ausência de aberturas laterais em geral apresentaram os melhores resultados para as variáveis analisadas, faz-se necessário a avaliação do efeito deste fator no desenvolvimento das árvores em campo, uma vez que estudos demonstraram que a utilização de recipientes com aberturas laterais promoveram poda aérea de raízes, gerando melhor morfologia de raízes nas mudas (SUNG e HAYWOOD, 2016) e após o transplante em campo (MARLER e MUSSER, 2016).

\section{CONCLUSÃO}

A utilização de métodos de produção de mudas de Pinus maximinoi via enraizamento de miniestacas e via germinação de sementes produziram mudas morfologicamente distintas, sendo que mudas produzidas por enraizamento de miniestacas apresentaram superioridade em altura, diâmetro, peso seco aéreo e peso seco total, enquanto mudas produzidas via germinação de sementes apresentaram maior sobrevivência e maior número de raízes.

A utilização de recipientes maiores influencia positivamente no desenvolvimento de mudas de Pinus maximinoi, apresentando maior influência em mudas produzidas via enraizamento de miniestacas.

A presença de aberturas laterais nos recipientes de produção de mudas de Pinus maximinoi afeta a maioria dos parâmetros morfológicos avaliados, apresentando em geral valores inferiores quando comparados à ausência de aberturas laterais.

Para produção de mudas de Pinus maximinoi via germinação de sementes ou enraizamento de miniestacas sugere-se a utilização de recipientes de $115 \mathrm{~cm}^{3} \mathrm{com}$ ausência de aberturas laterais. Recomenda-se também avaliar o efeito dos tratamentos no crescimento das árvores de Pinus maximinoi após plantio em campo.

\section{AGRADECIMENTOS}

Agradecemos à empresa KLABIN S/A pela disponibilização do viveiro de pesquisa, recursos e pessoal para realização do presente estudo.

\section{REFERÊNCIAS}

BAYLEY, A. D.; KIETZKA, J. W. Stock quality and field performance of Pinus patula seedlings produced under two nursery growing regimes during seven different nursery production periods. New Forests 13: 337-352, 1996.

BONGA, J. M. A comparative evaluation of the application of somatic embryogenesis, rooting of cuttings, and organogenesis of conifers. Canadian Journal of Forest Research. 45: 379-383, 2015. Disponível em: www.nrcresearchpress.com/cjfr. DOI: dx.doi.org/10.1139/cjfr-2014-0360.

BORDIN, I.; NEVES, C.S.V.J.; AZEVEDO, M. C. B.; VIDAL, L. H. I. Desenvolvimento de mudas de aceroleira propagadas por estacas e sementes em solo compactado. Ciência Rural, Santa Maria, v.35, n.3, p.530-536, 2005.

CUMBIE, P. W.; ISIK, F.; LI, B; GOLDFARB, B. Effects of propagule type on genetic parameters of wood density and growth in a loblolly pine progeny test at ages 10 and 11 years. Tree Genetics \& Genomes 7:1147-1158, 2011. DOI 10.1007/s11295011-0402-6. 
DOBNER JÚNIOR, M. D,; TRAZZI; P. A.; HIGA, A. R. SEITZ, R. A. Influência do volume do tubere e do método de plantio no crescimento de um povoamento de Pinus taeda aos nove anos de idade. Scientia Florestalis, Volume 41, n. 97. P.7-14, Março de 2013. Disponível em: www.ipef.br/publicações/scientia.

DONALD, D. G. M. The effect of tray volume and spacing on the growth and development of Pinus radiata Seedlings. Suid-Afrikaanse Bostoutydskrif - nr. 162, September 1992.

DVORAK, W.S; GUTIÉRREZ, E.A.; GAPARE, W.J.; HODGE, G.R.; OSORIO, L.F.; BESTER,C.; KIKUTI, P. Pinus maximinoi. In: Conservation \& testing of tropical \& subtropical forest tree species. CAMCORE Cooperative, College of Natural Resources, NCSU. Raleigh, p. 107-127, 2000.

FERRAZ, A. V.; ENGEL, V.L. Efeito do tamanho de tubetes na qualidade de mudas de Jatobá (Hymenaea courbaril L. var. stilbocarpa (Hayne) Lee et Lang.), Ipêamarelo (Tabebuia chrysotricha (Mart. ex DC.) Sandl.) e Guarucaia (Parapiptadenia rigida (Benth.) Brenan). Revista Árvore, vol.35, n.3, pp.413-423, 2011.

FERREIRA, D. F. Sisvar: a Guide for its Bootstrap procedures in multiple comparisons. Ciência Agrotecnologia. [online]. v.38, n.2 [citado 2015-10-17], pp. 109-112, 2014. Disponível em: ISSN 1413-7054. DOI: http://dx.doi.org/10.1590/S1413-70542014000200001.

HIGA, R. C. V; WREGE, M. S.; RADIN, B.; BRAGA, H.; CAVIGLIONE, J. H.; BOGNOLA, I.; ROSOT, M. A. D.; GARRASTAZU, M. C.; CARAMORI, P. H.; OLIVEIRA, Y. M. M. de. Zoneamento Climático: Pinus taeda no Sul do Brasil. Documentos 175. Embrapa Florestas. Colombo, PR, 2008.

IBÁ. Relatório IBÁ 2016. Industria Brasileira de Árvores. 2016. Disponível em: iba.org.br.

JELIC, G. Container size and soil preparation effects on afforestation success of one year old stone pine (Pinus pinea l.) seedlings in croatian mediterranenan area. 2014. Sumarski List,138 (9-10): 463-475, 2014.

JESUS, A. M. S.; CARVALHO, S. P.; SOARES, A. M.; Comparação entre sistemas radiculares de mudas de Coffea arabica $L$. obtidas por estaquia e por sementes. Coffee Science, Lavras, v. 1, n. 1, p. 14-20, abr./jun. 2006.

KLABIN. Dados históricos da estação meteorológica da KLABIN, Lagoa, Telêmaco Borba-PR, 2017.

LAMHAMEDI, M.; GIL, L.; HANK, M.; MARIO, R.; LINDA, V.; BERNIER, P. Y. Growth, physiology, and leachate losses in Picea glauca seedlings (1 0) grown in airslit containers under different irrigation regimes. Canadian Journal of Forest Research. vol:31 iss:11 pg:1968 -1980, 2001.

LU, P.; BELL, W.; CHARRETTE, P.; THOMPSON, M. Performance of jack pine (Pinus banksiana) rooted cuttings from proliferated dwarf shoots versus seedlings 8 years after planting. Canadian Journal of Forest Research. 42: 1404-1409, 2012. Disponível em: www.nrcresearchpress.com/cjfr. DOI:10.1139/X2012-079. 
MARLER, T.; MUSSER, C. Chemical and air pruning of roots influence posttransplant root traits of the critically endangered Serianthes nelsonii. Plant Root [1881-6754] Marler: v, 10 pg:21 -25, 2016.

MATOS, J. L. M., BONDUELLE, G. M. PRATA, J. G. IWAKIRI, S. Avaliação da qualidade de painéis compensados de Pinus maximinoi, Pinus oocarpa e Pinus tecunumannii com diferentes composições estruturais. Ciência Florestal, Santa Maria, v. 22, n. 2, p. 315-321, abr.-jun., 2012.

MITCHELL, R. G.; ZWOLINSKI J.; JONES N. B.; BAYLEY, A. D. Root volume and raising period affect field performance of Pinus patula cuttings in South Africa. Southern African Forestry Journal. n.204, July 2005.

MORA, F.; DEITOS, A.; ARNHOLD, E.; DANDOLINI, T. S. Análise do crescimento de árvores de sete anos de idade, originadas de estacas e sementes de Pinus radiata D. Don, provenientes de geração avançada de melhoramento. Revista do Instituto Florestal, v.17(2), pp.207-213. December 2005.

ORTEGA, U.; MAJADA. J; MENA-PETITE, A.; SANCHEZ-ZABALA. J.; RODRIGUEZ-ITURRIZAR, N.; TXARTERINA, K.; AZPITARTE, J.; ABEITIA, M. D. Field performance of Pinus radiata D. Don produced in nursery with different types of containers. New Forests (2006) 31:97-112 2006. DOI 10.1007/s11056-004-7364-6

PUÉRTOLAS J.; JACOBS, D. F.; BENITOA, L. F.; PENUELASA, J. L. Cost-benefit analysis of different container capacities and fertilization regimes in Pinus stock-type production for forest restoration in dry Mediterranean areas. Ecological Engineering. 44 210- 215. 2012. Disponível em: www.elsevier.com/locate/ecoleng DOI: http://dx.doi.org/10.1016/j.ecoleng.2012.04.005.

ROSE, R.; ATKINSON, M.; GLEASON, J.; SABIN, T. Root volume as a grading criterion to improve field performance of Douglas-fir seedling. New Forests. 5: 195209, 1991.

SUNG, S. J. S.; HAYWOOD, J. D. Air lateral root pruning affects longleaf pine seedling root system morphology. Proceedings: SCHWEITZER, CALLIE J.; CLATTERBUCK, WAYNE K.; OSWALT, CHRISTOPHER M., eds. 2016. Proceedings of the 18th biennial southern silvicultural research conference. eGen. Tech. Rep. SRS-212. Asheville, NC: U.S. Department of Agriculture, Forest Service, Southern Research Station. 614 p.

TSAKALDIMI, M. Prediction of planted seedling survival of five Mediterranean species based on initial seedling morphology New Forests, 44 (3): 327-339 MAY 2013. 